\title{
(Big) data in urban design practice: supporting high-level design tasks using a visualization of human movement data from smartphones
}

\author{
Angela Rout and Wesley Willett
}

We present the SmartCampus visualization tool, representing spatiotemporal data of over 200 student pathways and restpoints on a university campus. Based on our experiences with SmartCampus, we also propose a task-based framework that describes how practicing urban designers (specifically, architects) can use human movement data visualizations in their work. Although extensive amounts of location data are produced daily by smartphones, existing geospatial tools are not customized to specifically support high-level urban design tasks. To help identify opportunities in urban design for visualizing human movement data from devices such as smartphones, we used our SmartCampus prototype to facilitate a series of 3 participatory design sessions ( 3 participants), a targeted online survey (14 participants), and semi-structured interviews ( 6 participants) with architectural experts. Our findings showcase the need for location analysis tools tailored to concrete urban design practices, and also highlight opportunities for Smart City researchers interested in developing domain specific, visualization tools.

Key words: smartphone data, GPS, data visualization, architecture, urban design, task-based framework, high-level tasks,

\author{
Angela Rout \\ Department of Forest Resources Management, \\ University of British Columbia, Canada. \\ Email angela.rout@ubc.ca \\ Wesley Willett \\ Department of Computer Science, \\ University of Calgary, Canada
}




\subsection{Introduction}

Decades of research in using spatial data to inform design decisions has led to the development of a range of robust geographic visualization tools available to urban design practitioners. While this research is extensive, and Geographic Information Systems are widely available, new questions have emerged concerning how to present data from the increasing number of sensors, devices and technologies currently available. One abundant, yet still emerging, data resource is location data produced by smartphones. Since phones are carried by billions of people throughout their day, the data can be used to understand relationships between environments and human behaviors at a scale that was not previously possible. While existing geospatial visualizations enable designers to analyze and visualize physical features of landscapes, there are few (if any) data visualization tools that allow designers to readily explore the complex relationships between people and environments captured in large human movement datasets, from devices like smartphones. Since such tools are still emerging, many urban design practitioners may not yet know how to incorporate these kinds of data in their workflows. Meanwhile, developers creating new visualization and analysis tools lack a clear sense of how new platforms can best support the goals of urban designers - despite the fact that this kind of understanding is critical for good visualization design (Lam et al. 2012).

To help build tools that align with user needs and practices, researchers in the visualization community often use task-based frameworks to reason about the types of tasks that their target users may perform when using visualizations. Early task taxonomies, like Amar et al.'s (2005) were broad, identifying low-level tasks related to retrieving and understanding data and fundamental tasks for data visualization systems (Amar and Stasko 2004). These initial frameworks focused on tasks necessary to bridge 'analytic gaps' or difficulties faced when using data to make complex decisions. More recent task taxonomies have described user engagement (Mahyar et al. 2015), the relationship between interaction and inquiry (Pike et al. 2009), and spatio-temporal data visualizations (Andrienko et al. 2011). Researchers have focused on adapting task-based taxonomies for domain specific working cultures and expectations. For example, Bowes et al. (2018) propose a taxonomy for using spatio-temporal data in transportation and planning fields.

Task-based frameworks like these typically identify both high and low level tasks that a visualization might support (Amar and Stasko 2004). High-level tasks are complex and involve synthesis or evaluation of multiple pieces of information. They often involve applying information to real-world, domain-specific problems. Meanwhile, low-level tasks are typically simpler cognitive operations that involve altering or extracting information from a visualization. Describing the tasks performed within specific domains, as well as understanding real-world processes is a key part of data visualization research focused on developing tools that support professional objectives. 


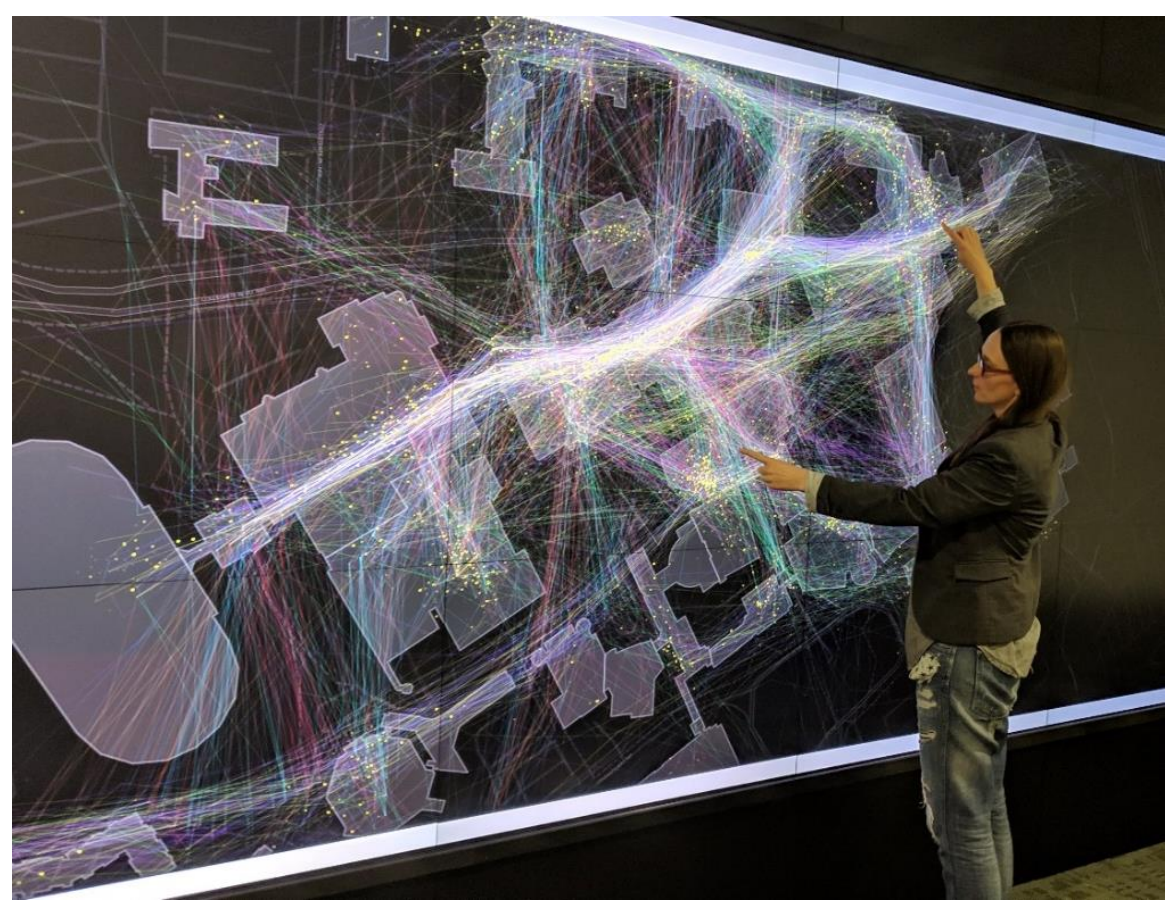

Fig. 1 We developed the SmartCampus visualization tool that allows urban designers to visualize student walking patterns across the university campus

We focus our attention specifically on the tasks urban design experts are likely to encounter when engaging with large-scale human movement data. While some existing GIS applications support smartphone data visualizations in urban applications, little work has examined the extent to which smartphone visualizations can be used within the existing practices of urban design professions. Instead, researchers have generally used visualizations themselves, or have presented methods for analyzing or visualizing the data. For example researchers have used location data to identify public amenities used by cyclists (Heesch and Langdon 2016), railroad crossings (Hudson et al. 2012), locations of congestion (Siljanoska and Korobar 2017), and cultural heritage sites of potential importance (Rolando and Scandiffio 2013). Visualizations of urban human movement data from smartphones have used a variety of methods including Kwan's space-time cube (Yun and Park 2014), 3D visualizations of individuals' spatio-temporal data (Wiehe et al. 2008; Zhang et al. 2017), or interactive displays of paths taken by cyclists (Pettit et al. 2016).

Here, we contribute the SmartCampus visualization tool, a visualization system (Fig. 1) that lets practitioners explore a large, longitudinal human movement dataset collected from smartphones. Using multiple research methods, we examined how such a tool would facilitate domain specific tasks. For all sections of our study, we 
worked with experts in the domain of architecture but the tasks we identify have application for any design profession that modifies urban environments at the pedestrian scale. For the development of the tool, we worked together with architects who had frequent, end-to-end involvement throughout the design of the tool. Our process involved three face to face sessions, with feedback on iterative tool designs. We also enlisted 14 architectural design experts to provide feedback on the SmartCampus visualization tool and the tasks that it can support, via an online survey. Lastly, we conducted six semi-structured interviews to explore the realities of using data visualizations in design practice. Based on these experiences we present a framework for design tasks that are supported by smartphone data. We then describe in detail, five, high-level design tasks and their role in real-world design offices. Lastly, we also outline considerations for future tool developers when seeking to use smartphone data visualizations to support these tasks. These contributions will enable future smart city researchers to develop geospatial data visualizations that focus on the specific uses of new, big data resources in the day-to-day practice of urban design professionals.

\subsection{Methodology}

Our study was informed by these underlying research questions: 1) What high-level design tasks do architectural design experts perform that have potential to be supported by new visualization tools? 2) How important are these high-level tasks to good design, and where to they sit within real-world architecture practice process? 3) In what specific ways might visualization be used to accomplish these tasks?

\subsection{The smartphone data}

To answer our research questions, we built a visualization tool that visually displays a large dataset collected from 300 university students (Fig.1, smartcampus.ucalgary.ca/vis/) We approached students in the central student union building and asked if they are willing to share their personal location histories. To maintain some privacy, other demographic data was not collected. Thus, we are not able to compare our sample with characteristics of the general student population. Yet, the student union building is central and visited by a cross section of students from across the campus. Students each downloaded and shared their entire location history using the Google Takeout service on Android phones, which allows users to retrieve copies of personal data hosted by the company. Since individuals may turn location services off periodically, may not always connect with internet services, or may have recently purchased their phone, there is a large variability in the data collected among students. This results in a dataset that has fewer points than is expected if all participants contributed data over the full timespan (2012-2017). After cropping to 
locations on campus (before processing) the data includes 2 million discrete spatiotemporal data points and was collected in June 2017. Each participant's data included a unique User ID along with a sequence of coordinates and timestamps documenting their smartphone's position over time at intervals as frequent as one minute. We obtained explicit consent from participants and provided participants with a $\$ 25$ gift card incentive. This research was approved by our university research ethics board.

During data preprocessing, we used several techniques to preserve the privacy of individual participants. We first removed all data from outside the university campus, then segmented the data into smaller sub-paths and removed unique identifiers that would make it possible to identify individuals' daily routines. The resulting dataset can be used to observe flows of movement for multiple individuals, but there is no way to isolate a single user.

In addition to addressing privacy issues, much of our data preprocessing involved improving the data quality. Other studies indicate that GPS data from smartphones can vary considerably (ranging from about $8 \mathrm{~m}$ on average to as inaccurate as $18 \mathrm{~m}$ (Lee et al. 2017)). Based on our spot-checks, accuracy for our data likely falls within a similar range. When visualizing and exploring our data we found no evidence that the data had been snapped to existing pathways or features. Our first intention was to visualize raw data, although we found there was so much noise, we could not visually discern clear patterns, and thus the data required at least minimal processing. To avoid excessive time spent on data cleaning we chose to use a simple processing algorithm that was easily interpreted by our participants. Other sophisticated processing and cleaning approaches are available (Kerr et al. 2011; Idrissov and Nascimento 2012; Pettit et al. 2016; Vementala et al. 2017). Our method is publicly shared through our website (smartcampus.ucalgary.ca).

The method we used involved grouping sets of points from individuals which were spatially (radius of $50 \mathrm{~m}$ ) and temporally (at least 10 minutes) clustered into single spatial points that we called restpoints. This term differs from what Pérez-Torres et al. (2016) refer to as a "stay point", in that a restpoint represents a single visit, and a stay point represents locations that are frequently visited. Second, we linearly connected consecutive unprocessed points that followed a spatial trajectory within a reasonable timeframe (between $15 \mathrm{~m}$ and $200 \mathrm{~m}$ in 20 minutes), into discrete paths. To create these tolerances, we iterated multiple variations of spatial and temporal settings using data from our research team. We visually assessed whether points represented real movements and aimed to retain as much data as possible. The final set of points and paths were significantly smaller than our raw data files. This is because our restpoints amalgamate multiple points into a single location with a duration parameter. From our visual assessment, we were satisfied that clustered 


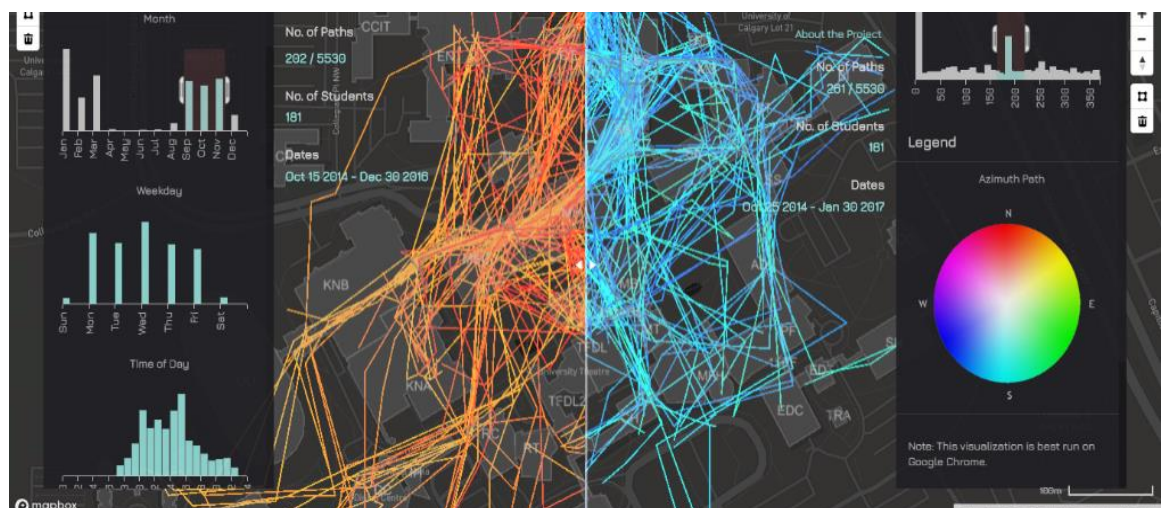

Fig. 2 The SmartCampus visualization tool has multiple features including filters for time of year, week and day as well as weather variables such as temperature. The tool uses color to represent movement direction and includes a slider to allow for users to compare two different filter settings

points and paths were generally similar to the movement and resting patterns of our team member and were significantly improved over representations of the raw data, although further research is needed to quantify the improvement. After our preprocessing of the entire data set, we removed $\sim 100$ participants who did not provide enough clustered data (either due to data gaps or an original small number of points) to satisfy our tolerances. Thus, the SmartCampus visualization tool represents data from just over 200 students (mean number of points per student $=146, \mathrm{sd}=78$ ).

\subsection{Visualization design}

The SmartCampus visualization tool displays the complete dataset of paths and restpoints, spatially cropped to the university campus and superimposed over a basemap showing walkways, landscape features and building footprints. Features of the SmartCampus visualization tool allow designers to filter data by time, identify the movement direction of traffic, and select locations of interest (Fig. 2). Urban designers can filter temporally to show specific months, days of the week, and times of the day using filtering buttons and interactive distribution charts. They can also select geographic locations using an interactive polygon selection tool. We used colour to visualize direction of movement so that architects could easily differentiate traffic flows in and out of locations of interest. We also included a two-up interface that makes it possible to compare two different filtering options. The SmartCampus visualization tool, aggregated data, and source code are all publicly available (smartcampus.ucalgary.ca). 


\subsubsection{Participatory sessions}

Throughout the duration of the project, we repeatedly engaged in participatory focus groups with a team of three practicing architects who were actively involved in a large-scale architectural project that was reshaping one of the core areas of the campus (Fig. 3). We ran 3 two-hour sessions with the same architects, distributed evenly throughout our four-month design cycle. During these sessions, the architects used the tool to examine student movement in relevant areas of campus and discussed those observations in the context of their active project. Although the overall number of participants was small, their expertise and the repeated experiences with the data and our evolving tool allowed them to provide multiple rounds of deep and insightful feedback which directly impacted both the designs and our conceptual framework. Such approaches have been described and supported in other data visualization research (Rieman 2002; Saraiya et al. 2006).

When developing our framework, we first reviewed notes and audio recordings collected during the first two focus groups to generate a list of 56 questions that the architects asked while examining the data and our initial visualizations. We then sorted the questions into task-centric groups. At each phase, we presented our synthesis back to the architects for further feedback and to ensure our findings matched their perceptions. Most questions corresponded to low-level tasks such as identifying patterns in times and locations of activities. These included questions like "what

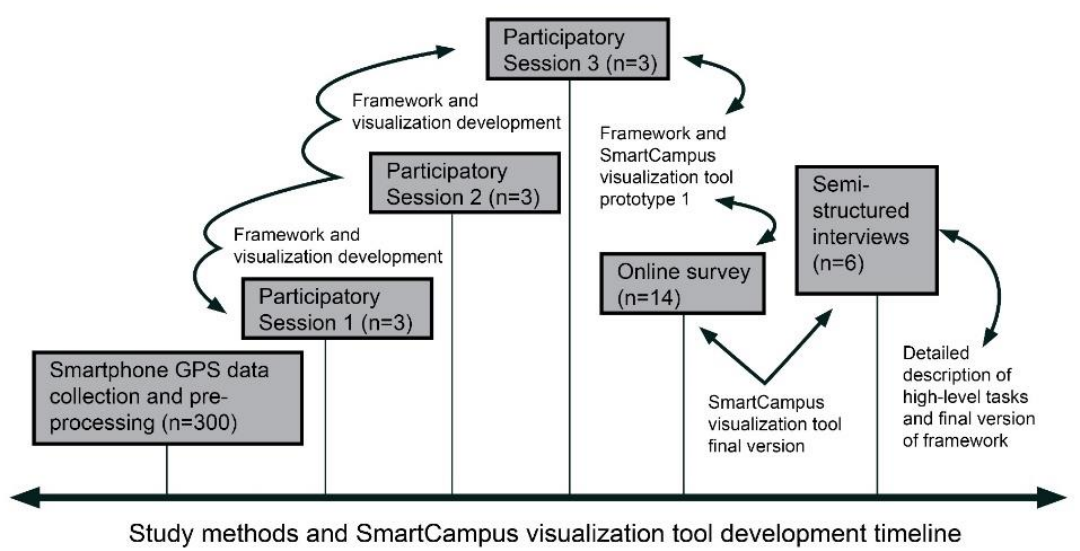

Fig. 3 Timeline indicating the sequence of prototype and framework development over our three study methods 
framework that included high- and low-level design tasks that may be supported by data visualization tools. We shared this framework with the participants, and modified it based on their feedback.

One outcome of the participatory sessions was a draft task-based framework that we slightly modified after subsequent interviews. We present the final framework here (Table 1). The low-level tasks build on the relationships described by Andrienko et al. (2011). We list cognitive tasks, significant places, significant times and movement behaviours, and use them to generate low-level tasks for visualizing human movement data. We selected cognitive tasks by identifying the appropriate tasks for spatio-temporal visualizations, starting from Amar et al. (2005)'s taskbased taxonomy. Cognitive tasks, behaviour, location, and times can be combined to form a low-level task, for example: compare differences between people walking indoors versus outdoors at noon. For an urban designer to use a visualization of human movement data to accomplish this task, we expect they would filter the data by time, and visually compare indoor and outdoor trajectories.

We then used an online survey to assess the relevance and utility of the framework for real-world design tasks. The survey included registered architects $(n=7)$, intern architects $(n=3)$, architectural students $(n=2)$, and graduates of an architectural graduate degree $(n=2)$ (Table 2$)$. We asked survey respondents how important each of the tasks in the framework were to their own practice. We then asked participants to use the SmartCampus visualization tool to accomplish a selection of low-level tasks. After they had spent time exploring the data, we asked participants whether the SmartCampus visualization tool was an improvement over the current tools they use. Smartphone data visualization tools are not commonly available for architects, but other tools that may accomplish similar tasks may include: GIS mapping, hand drawn maps or digitally illustrated maps representing data from site visits, surveys, observations, or census.

Lastly, building on our findings from the online survey and the participatory sessions, we conducted six semi-structured interviews with architecture experts. For these interviews we used snowball sampling to recruit architects, starting with the professionals in our contact list. Our semi-structured interviews included registered architects $(\mathrm{n}=1)$, intern architects $(\mathrm{n}=3)$, an industry expert without architecture training $(n=1)$, and a graduate of a graduate architecture program $(n=1)$ (Table 2). Based on feedback from the architects, we introduced a feature that allowed them to easily compare two different filtered settings and included a geographic selection tool that allows them filter data by location. During the interviews we gave a short 
Table $1 \mathrm{~A}$ task-based framework for visualizing human movement data for urban design applications. Low-level tasks combine user cognitive tasks with behaviours, locations and times. Highlevel tasks address real-world design activities that visualizations of human movement data can potentially support.

\section{Task-based framework for visualizing human movement data for urban design applications}

\section{High-level urban design tasks:}

Deciding locations that optimize opportunities for a design intervention

Analyzing factors that affect how people behave in a space

Understanding the flow of human movement in a space, over time

Empathizing with pedestrian/human experiences of an environment

Cultivating inspiration to generate new ideas and solutions to problems

\section{Low-level urban design task matrix:}

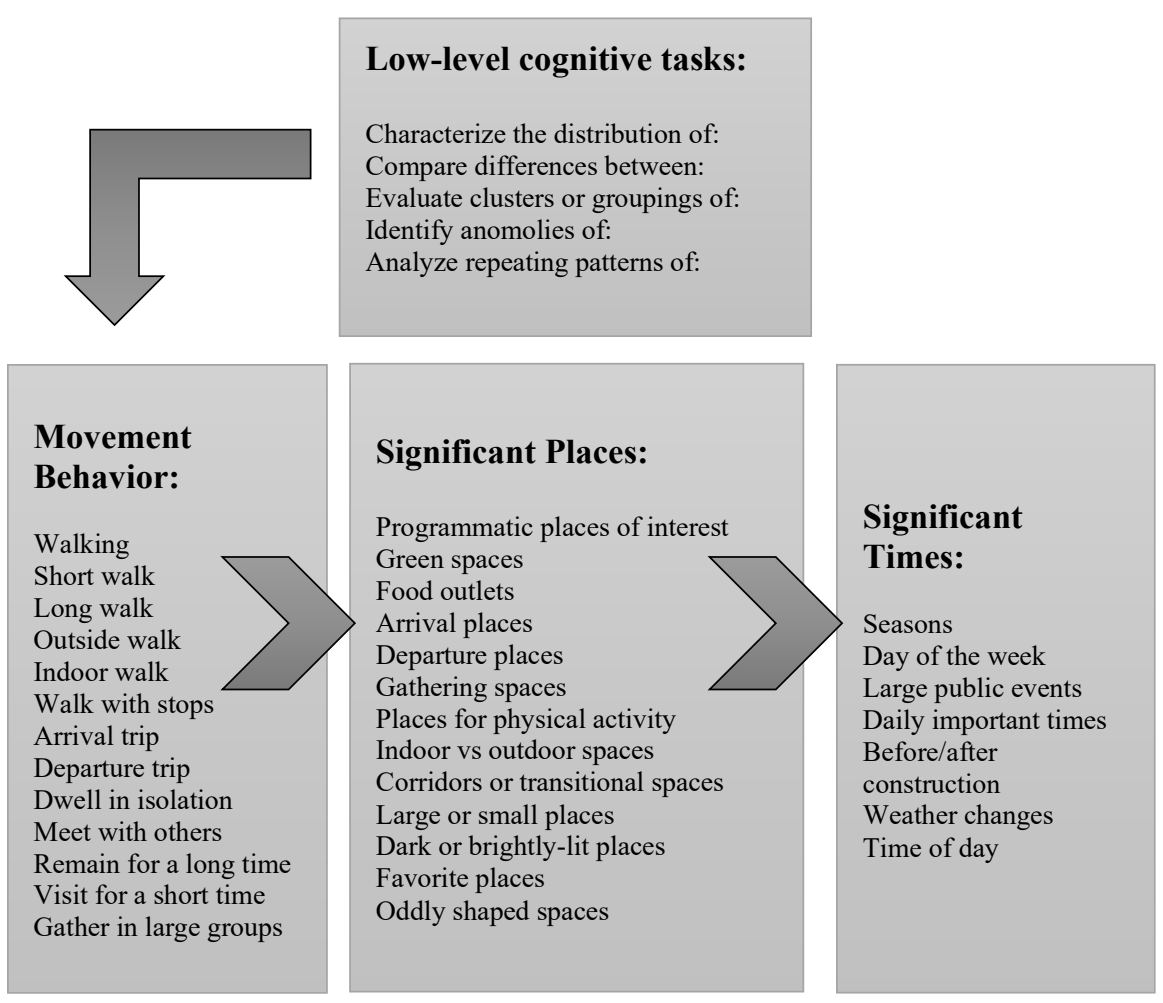


Table 2 The professional roles of our study participants.

\begin{tabular}{|c|c|c|c|}
\hline & Participatory Sessions & $\begin{array}{c}\text { Online } \\
\text { Survey }\end{array}$ & Interviews \\
\hline Registered Architects & $\begin{array}{c}\text { The same 3 architects } \\
\text { attended all 3 participa- } \\
\text { tory sessions }\end{array}$ & 7 & 1 \\
\hline Intern Architects & & 3 & 3 \\
\hline Architecture students & & 2 & 1 \\
\hline $\begin{array}{c}\text { Architecture degree } \\
\text { holders }\end{array}$ & & 2 & 1 \\
\hline Industry experts & & & \\
\hline
\end{tabular}

is the peak time for transit use in fall?" A few questions were indicative of higherlevel thinking. For example: "what is the best place on campus for a new design intervention?" Using these questions as a guide, we developed a draft task-based presentation introducing the SmartCampus visualization tool. We demonstrated the tool features and described the smartphone data collection process. The interviews then involved a series of questions about high-level design tasks. We transcribed and coded answers from the semi-structured interviews and integrated them with findings from our previous phases. In the discussion section of this paper, we report the concepts and themes that were shared among participants.

\subsection{Results}

Through analysis of responses, we identified five high-level design tasks. These are listed in Table 1: (1) Identifying locations that optimize opportunities for design intervention. (2) Analyzing factors that affect how people behave in a space. (3) Understanding the flow of human movement over time. (4) Empathizing with pedestrian/human experiences of an environment. (5) Cultivating inspiration to generate new ideas and solutions to problems. We identified the first four design tasks based on our initial participatory workshops and included questions about them on the online survey. Almost all respondents to the survey agreed that the first four high-level architectural design tasks were an important part of their design process (Fig. 4). Most survey respondents also felt that the SmartCampus visualization tool supported these tasks better than their current toolsets. One participant commented that they had used Space Syntax (Ratti 2004) in the past and stated that the base layers were easier to understand in that system, particularly when identifying elevation information. The fifth high-level emerged later, based on feedback from the survey and the semi-structured interviews. 
These tasks are important for me to create successful designs:

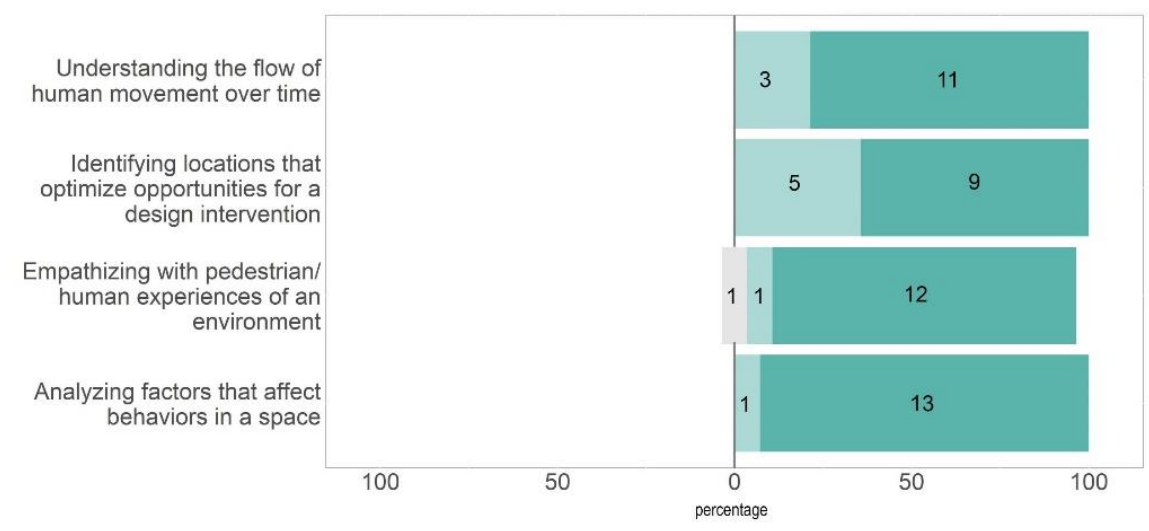

The SmartCampus visualization tool supports these tasks better than my current tools:

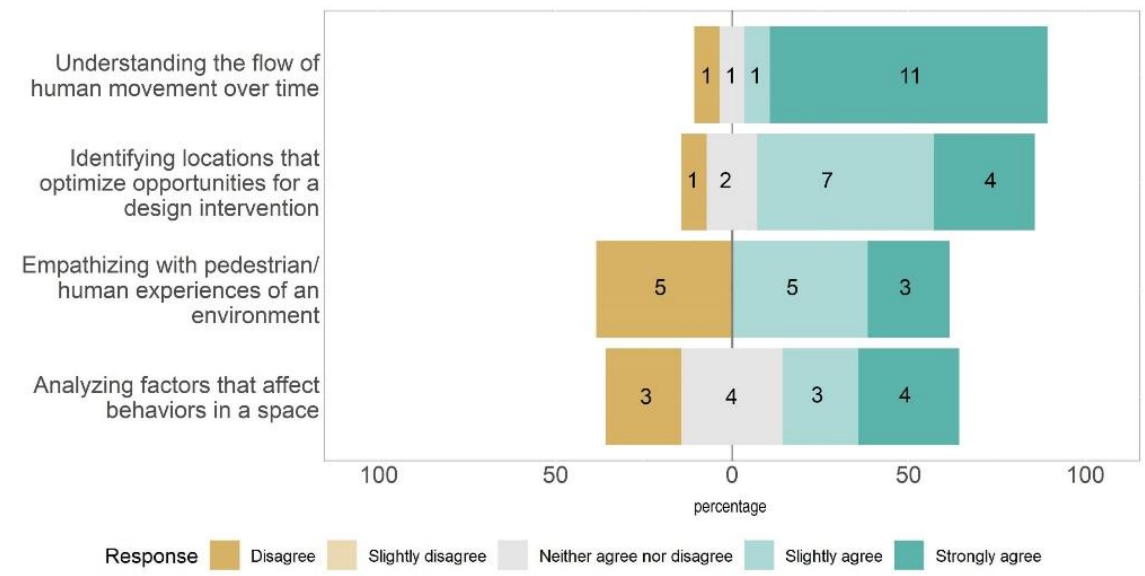

Fig. 4 Responses to the online survey $(\mathrm{n}=14)$ questions. Top-All (except one) participants agreed or strongly agreed that all of the high-level design tasks we identified are important parts of their successful design practice. Bottom-Almost all participants (11/14) strongly agreed that the SmartCampus visualization was better at helping them understand the flow of human movement over time than their current tools 
The urban designers we spoke to also highlighted numerous concrete ways in which human movement data visualizations could improve their ability to perform these tasks efficiently and accurately. In particular, respondents saw opportunities for visualization tools to help them identify locations that optimize opportunities for design intervention (Task 1) and understand the flow of human movement over time (Task 3).

\subsection{Discussion}

Much like our survey respondents, all six participants in our semi-structured interviews agreed that the high-level tasks we identified have profound importance across many different stages of the architectural design process - including for predesign, conceptual design, schematic design, design development, construction documents, contract admin, and post occupancy. Based on their feedback, we discuss specific opportunities for data visualizations to support each task and make recommendations for future tool developers.

\subsection{Identifying locations that optimize opportunities for design intervention}

When performing this task, designers typically use multiple sources of information to select the best location for a new design or feature. This entails consolidating, evaluating, and balancing information about how people use a site, then using that information to select the best location for the design intervention. Such interventions may include situating a building on a site, deciding the location for programmatic elements (such as a new coffee shop or study nook), or deciding where to place specific design features (like windows or benches).

All interview participants reported that this task typically occurs near the beginning of their practice, during predesign and conceptual design. However, participants P2 and P6 also noted that some smaller design decisions related to this task such as locating benches may fall later in the design process during schematic design.

Opportunities: Tools may be able to identify outliers, or instances where human behavior is unusual, which could indicate an opportunity for a new design intervention. For example, a visualization of paths that converge at a door or hallway may indicate unwanted congestion, and an opportunity for better design. We also noted that smartphone location data has the potential to represent multiple human behaviours that, when displayed over the contextual map, may signify potential opportunities for design. For example, smartphone location data could be used to identify behaviours such as socializing, commuting, eating lunch or taking recreational 
walks. Knowing when and where these activities occur may help designers select new design features or locate programming. To support this task new tools should investigate how to group, analyze and represent these movement behaviours.

\subsection{Analyzing factors that affect how people behave in a space}

Designers engaged in this task explore the relationship between designed environments and human behaviours. This typically involves observing people's interactions with design elements over time. It requires designers to study people in various conditions, make hypotheses as to why people behave a certain way, and test assumptions based on subsequent observations. For example, a designer may observe people near a public fountain in the data. They could filter the data by different times of day, and make a hypothesis that people usually stand near fountains in the morning. They could then observe other fountains in different locations, or at different times of the year, to see if their hypothesis holds true.

Three interview participants (P2,P3, and P6) felt that the most obvious stage for analyzing the effects of design on people is at post-occupancy, where they could examine the impact of their own choices on human behavior. However, two participants (P4 and P5) mentioned engaging in this task throughout the design phase. All interview participants expressed a strong desire to use data visualizations to assess their designs and to understand how well their designs performed in terms of human behavior. Interview participants P2, P5 and P6 called out specifically how the SmartCampus visualization tool supported this task by indicating locations where people lingered for extended periods.

Opportunities: The GPS signals that smartphones rely on for most location estimates are often blocked by urban infrastructure such as ceilings and tall buildings. Additionally, smartphone location data alone does not reliably indicate altitude and thus can be unreliable for characterizing movement in indoor spaces, particularly within multi-story buildings. As a result, location data from a small number of smartphones is often too imprecise to provide a clear picture of human movement relative to low-level architectural features like walls and rooms. In our tool, despite only minimal processing, in locations where there are many paths, it is possible to visually discern the "mean" flow of traffic that follows indoor hallways and routes. This suggests that in cases where there is a lot of data available, it may be possible to indicate detailed indoor traffic flows using existing, inaccurate smartphone localization. In our visual assessments of the tool, this visual "mean" was not obvious with the restpoints. Yet, new location positioning methods are increasingly improving, and therefore thus, visualizing indoor spaces with smartphone data may greatly improve in the near future (Kang and Han 2015; Maghdid et al. 2016). 
When developing the SmartCampus visualization tool, we incorporated some basic strategies to reduce the impact of noisy data, including isolating paths that likely represented continuous walking and restpoints where individuals remained for ten minutes or more. Going forward, more active aggregation of data across users, locations, and times could also be used to identify important locations and routes. Architects in our participatory design sessions expressed interest in this task. In response we added a two-up interface to our tool. This interface allows users to visually compare two different filtering settings. This tool supports this high-level design task because designers can more easily compare paths in a similar location at two different times (before or after new construction) or is various weather conditions, thus allowing them to observe the impact of changes in time and weather on paths people use.

\subsection{Understanding the flow of human movement over time}

Designers performing this task seek to learn about the overall direction and rate of human movement over a site, as well as changes in those behavior across days, weeks, and seasons. Understanding human movement flows on a site helps architects consciously decide how their new design will either facilitate or interrupt existing human movement patterns.

Several interview participants $(\mathrm{P} 1, \mathrm{P} 2, \mathrm{P} 6)$ mentioned that this task usually takes place early during the conceptual or schematic design phases, while P5 suggested it could also be performed post-occupancy. When using the SmartCampus tool, three interview participants (P2, P3 and P5) remarked at how the visualization could help them characterize building entrances, while four participants (P1,P4,P5 and P6) discussed how the data helped explain seasonal and weather-related changes in campus use. The system also prompted Participants 2 and 6 to ask entirely new questions wondering whether campus interventions could be designed to behave differently in the morning versus the afternoon, or if design elements could be easily relocated based on future changes in traffic patterns. Four interviewees (P2, P3, P5, P6) talked about how understanding people's movement across their site helped them identify how their design might build upon popular existing routes.

Opportunities: In our survey, three architects mentioned that - although the visualizations showed distinct traffic and movement patterns - they were unsure how much they could rely on the data to make interpretations. Similarly, when presenting the data to architects we observed that their interpretations and generalizations sometimes relied on inaccurate assumptions about the completeness and accuracy of the data. This challenge manifested itself in three forms: (1) confusion about what population the human movement data visualization could be generalized to, (2) over-interpretation of a small number of paths or points as representative of the data we collected and (3) uncertainty about how spatially precise the data was. In 




a)

b)
When more paths are visible, subtle movment patterns can be observed

Individual paths may cross buildings where there are no doors or hallways

Fig. 5 In some locations, where many paths overlapped, the mean route of travel can be easily visually discerned b) Despite extensive pre-processing, in some cases the data retained a low-level of spatial precision, resulting in obvious errors such as routes that cross buildings where there are no doors

locations where there is a lot of data clustered or overlapped, clear patterns are discernable. This applied to our visualization where paths are not snapped to a grid, and therefor all paths are slightly different, thus creating a way to visually discern the "mean" route taken by many individuals. This effect of generating a "mean" with multiple paths is one way that our visualization can accommodate some of the error produced by noisy data. As such, the interpretability may be best suited for overall flows of people, rather than specific locations (Fig. 5). In our tool we attempted to address potential misconceptions by providing a tutorial video and dynamic participant counts directly in the visualization. However, further research is needed to explore the severity of these kinds of misinterpretations and how visualization designs can help to pre-empt them.

\subsection{Empathizing with pedestrian/human experiences of an environment}

This task occurs when a designer puts themselves into an end-user's shoes to understand what it feels like to use the places they design. To perform this task, designers observe where many people spend time on a site to imagine or envision those individuals' experience of the location.

Participants P2 and P5 described performing this task early in the design process during conceptual or schematic design. However, interview participants P3 and P5 discussed how thinking about end-user experiences can occur even near the end of design development, when material finishes, furniture and interior elements are being selected. Two interview participants (P4 and P6) specifically observed that visualizing restpoints could directly support this task, helping designers choose distinct starting points from which to identify anomalies or problems in a design. 
Meanwhile, four interview participants (P2, P3, P4, P5) commented that too often designers make decisions based solely on their own experience, and any data at all could help them check their assumptions, or at least remind them to think of others. Participant P2 felt strongly about using data to examine spaces from a variety of individual perspectives - especially now, when diversity and inclusion have come to the forefront of conscientious design practice (Heylighen et al. 2017).

Opportunities: Future tools should provide mechanisms to help designers to envision themselves within a space, or to see location and movement data in context. We observed that locations that were represented in the base layer map of our prototype tool, including labels of buildings, paths, trees, and other qualitative information about the environment, impacted the ability of the architects to make highlevel interpretations of the data. This was confirmed in our questionnaire, where four respondents stated that it was difficult to interpret our data visualization unless they were familiar with the campus environment. Future systems may be able to provide more of this kind of context by including realistic building models or streetlevel imagery, or even by allowing on-site exploration via augmented reality. Other datasets that might help designers empathize with end-users include comments or direct quotations from individuals who frequent the site, or amenity data such as the locations of shops, benches, trees or other key design elements.

\subsection{Cultivating inspiration to generate new ideas and solutions to problems}

While creativity and problem-solving are critical throughout all phases of a design project, our interview participants highlighted how ideating to develop new formal or spatial design ideas can itself constitute an important and discrete design task - and one in which visualization tools can play a role. Three interview participants (P2, P4 and P6) described explicit site-oriented ideation during early-stage design, a practice which P6 framed in terms of "creating a story around a site". This inspiration process involves finding linkages between quantitative and qualitative data stories to develop an overall design strategy, then using those stories to encourage inspiration and new design solutions. Participants P1, P2, P3 and P6 all stressed the potential for visualizations to support this practice where existing patterns of use could serve as inspiration for interventions or alternative designs. P3 felt that the diagrammatic character of the paths in our visualization gave them inspiration for new aesthetic strategies.

Opportunities: Our discussions related to this final task imply that the visual elements of new visualization tools may give urban designers jumping-off points for the development of new formal approaches. Although, to what degree this is true, 
and the impact of different visualization methods on a designer's ability to generate new ideas, requires further research.

\subsection{Conclusion}

Big data is poised to revolutionize urban design, yet few tools necessary to incorporate that data deeply into the design process remain relatively nascent. Our prototype visualization tool illustrates the potential of these approaches, supporting rich interactive exploration of data from over 200 student smartphones on a university campus. Meanwhile, our domain specific task-based framework outlines five highlevel design tasks in urban design that present promising opportunities for future tools. Urban designers' experiences with our prototype (including six interviews, an online survey, and three participatory sessions with architects) suggests real enthusiasm for visualizations that can integrate into key steps in designers' workflows - helping, them observe and understand existing usage patterns and allowing them to evaluate potential designs.

As an increasing amount of data is being produced by digital devices it is necessary for researchers to develop methods for practitioners to access and utilize them to make impactful decisions. Other potentially rich data sources that are complimentary to smartphone location data include wifi log-ins, calendar events, building energy use, data from motion sensors and transit ticket sales. In addition, other data that describes the spatio-temporal context, such as weather, calendar dates of large events, and spatial design features can enhance visualizations of location data. We recommend that future researchers explore methods for representing noisy data, for supporting sound interpretations and for supporting high-level design tasks and using visualizations of human movement data. We also recommend that developers of new visualization tools consider the high and low-level design tasks of practitioners whose decisions impact urban form.

\section{Acknowledgments}

Funded by the Taylor Family Digital Library: Academic Research \& University Libraries Andrew W. Mellon Foundation Subgrant. University of Calgary

Development team: Alec McAllister and Teresa Van 
Amar R, Eagan J, Stasko J (2005) Low-level components of analytic activity in information visualization. In: Proceedings - IEEE Symposium on Information Visualization, INFO VIS

Amar R, Stasko J (2004) A knowledge task-based framework for design and evaluation of information visualizations. In: Proceedings - IEEE Symposium on Information Visualization, INFO VIS

Andrienko G, Andrienko N, Bak P, et al (2011) A conceptual framework and taxonomy of techniques for analyzing movement. $\mathrm{J}$ Vis Lang Comput. https://doi.org/10.1016/j.jvlc.2011.02.003

Bowes J, Sara D, Juneja M, et al (2018) User-Centered Taxonomy for Urban Transportation Applications. In: In International Conference on HCI in Business, Government, and Organizations. Springer, pp 577-593

Heesch KC, Langdon M (2016) The usefulness of GPS bicycle tracking data for evaluating the impact of infrastructure change on cycling behaviour. Heal Promot J Aust 27:222-229. https://doi.org/10.1071/HE16032

Heylighen A, Van der Linden V, Van Steenwinkel I (2017) Ten questions concerning inclusive design of the built environment. Build Environ. https://doi.org/10.1016/j.buildenv.2016.12.008

Hudson JG, Duthie JC, Rathod YK, et al (2012) Using smartphones to collect bicycle travel data in Texas. Texas Transportation Institute

Idrissov A, Nascimento MA (2012) A Trajectory Cleaning Framework for Trajectory Clustering. Mob Data Chall 2012 (by Nokia)

Kang W, Han Y (2015) SmartPDR: Smartphone-based pedestrian dead reckoning for indoor localization. IEEE Sens J. https://doi.org/10.1109/JSEN.2014.2382568

Kerr J, Duncan S, Schipperjin J (2011) Using global positioning systems in health research: A practical approach to data collection and processing. Am J Prev Med 41:532-540. https://doi.org/10.1016/j.amepre.2011.07.017

Lam H, Bertini E, Isenberg P, et al (2012) Empirical studies in information visualization: Seven scenarios. IEEE Trans. Vis. Comput. Graph. 18:1520-1536

Lee L, Jones M, Ridenour GS, et al (2017) Comparison of accuracy and precision of GPS-enabled mobile devices. In: Proceedings - 2016 16th IEEE International Conference on Computer and Information Technology, CIT 2016, 2016 6th International Symposium on Cloud and Service Computing, IEEE SC2 2016 and 2016 International Symposium on Security and Privacy in Social Netwo

Maghdid HS, Lami IA, Ghafoor KZ, Lloret J (2016) Seamless Outdoors-Indoors Localization Solutions on Smartphones: Implementation and Challenges. ACM Comput Surv. https://doi.org/10.1145/2871166

Mahyar N, Kim S, Kwon BC (2015) Towards a taxonomy for evaluating user engagement in information visualization. In: In Workshop on Personal Visualization: Exploring Everyday Life. $\mathrm{p} 2$

Pérez-Torres R, Torres-Huitzil C, Galeana-Zapién H (2016) Full on-device stay points detection in smartphones for location-based mobile applications. Sensors (Switzerland) https://doi.org/10.3390/s16101693

Pettit CJ, Lieske SN, Leao SZ (2016) Big bicycle data processing: from personal data to urban applications. In: ISPRS Annals of the Photogrammetry, Remote Sensing and Spatial Information Sciences. Prague, pp 173-179

Pike WA, Stasko J, Chang R, O'Connell TA (2009) The science of interaction. Inf Vis. https://doi.org/10.1057/ivs.2009.22

Ratti C (2004) Space syntax: Some inconsistencies. Environ Plan B Plan Des. https://doi.org/10.1068/b3019

Rieman J (2002) A field study of exploratory learning strategies. ACM Trans Comput Interact. https://doi.org/10.1145/234526.234527

Rolando A, Scandiffio A (2013) Mobile applications as tool for exploiting cultural heritage in the region of turin and Milan. Int Arch Photogramm Remote Sens Spat Inf Sci - ISPRS Arch 40:525-529. https://doi.org/10.5194/isprsarchives-XL-5-W2-525-2013 
Saraiya P, North C, Lam V, Duca KA (2006) An insight-based longitudinal study of visual analytics. IEEE Trans Vis Comput Graph. https://doi.org/10.1109/TVCG.2006.85

Siljanoska J, Korobar VP (2017) Citizen involvement in planning process innovation: The case of centar municipality in Skopje . Prostor 25:87-97

Vementala N, Papotti P, Sarwat M (2017) A framework for interactive geospatial map cleaning using GPS trajectories. In: ACM International Conference Proceeding Series

Wiehe SE, Carroll AE, Liu GC, et al (2008) Using GPS-enabled cell phones to track the travel patterns of adolescents. Int J Health Geogr 7:. https://doi.org/10.1186/1476-072X-7-22

Yun HJ, Park MH (2014) Time-Space Movement of Festival Visitors in Rural Areas Using a Smart Phone Application. Asia Pacific J Tour Res 1665:1-20. https://doi.org/10.1080/10941665.2014.976581

Zhang L, Rong P, Xia H, et al (2017) Smartphone-based spatiotemporal behavior analysis on college students in Chinese newly developed campus: A case study for Henan university. In: International Conference on Geoinformatics 